\title{
ON FINITELY DOMINATED CW COMPLEXES
}

\author{
JERZY DYDAK
}

\begin{abstract}
Let $D$ be the class of all CW complexes homotopy dominated by finite $\mathrm{CW}$ complexes. In this paper we prove the following theorem.

THEOREM. Suppose a connected $C W$ complex $X \in \mathbb{D}$ is a union of two connected subcomplexes $X_{1}, X_{2}$ with $X_{1} \cap X_{2}=X_{0} \in \mathbb{D}$. Then $X_{1}, X_{2} \in \mathscr{D}$ if one of the following conditions is satisfied:

(i) $\pi_{1}\left(X_{0}, x\right) \rightarrow \pi_{1}(X, x)$ is a monomorphism for each $x \in X_{0}$

(ii) $\pi_{1}\left(X_{i}\right) \rightarrow \pi_{1}(X)$ is a monomorphism for $i=1,2$ and $\pi_{1}\left(X_{1}\right), \pi_{1}\left(X_{2}\right)$ are finitely presented.
\end{abstract}

1. Introduction. Let $\mathscr{D}$ be the class of all $\mathrm{CW}$ complexes homotopy dominated by finite CW complexes. In this note we discuss the following question: Suppose a connected CW complex $X$ is a union of two connected subcomplexes $X_{1}$ and $X_{2}$. Under what conditions does $X \in \mathscr{D}$ and $X_{1} \cap X_{2}=X_{0} \in \mathscr{D}$ imply that $X_{1}$, $X_{2} \in \mathscr{D}$ ?

In [4, p. 48] L. C. Siebenmann answered the above question positively in case where $\pi_{1}\left(X_{i}\right) \rightarrow \pi_{1}(X)$ has a left inverse for $i=1,2$ and asked (p. 49) whether the condition that $\pi_{1}\left(X_{i}\right) \rightarrow \pi_{1}(X)$ is a monomorphism, $i=1,2$, is sufficient for $X_{1}$ and $X_{2}$ to be in $\mathscr{D}$.

The following results give partial answers to Siebenmann's question.

1.1. TheOrem. Suppose a connected $C W$ complex $X \in \mathscr{D}$ is a union of two connected subcomplexes $X_{1}$ and $X_{2}$ with $X_{1} \cap X_{2}=X_{0} \in \mathscr{D}$. If, for $i=1,2$,

(i) $\pi_{1}\left(X_{i}\right) \rightarrow \pi_{1}(X)$ is a monomorphism and

(ii) $\pi_{1}\left(X_{i}\right)$ is finitely presented, then $X_{1}, X_{2} \in \mathscr{D}$.

1.2. TheOrem. Let $X \in \mathscr{D}$ satisfy the hypotheses of Theorem 1.1. If $\pi_{1}\left(X_{1}\right) \rightarrow$ $\pi_{1}(X)$ is a monomorphism, $\pi_{1}\left(X_{1}\right)$ is finitely presented and $X_{0}, X_{2} \in \mathscr{D}$, then $X_{1} \in \mathscr{Q}$.

1.3. Theorem. Let $X \in \mathscr{D}$ satisfy the hypotheses of Theorem 1.1. If $\pi_{1}\left(X_{0}\right) \rightarrow$ $\pi_{1}(X)$ is a monomorphism, then conditions (i) and (ii) of Theorem 1.1 hold. In particular, $X_{1}, X_{2} \in \mathscr{D}$.

Examples in [4, pp. 49, 83-89] show that some restriction on fundamental groups is necessary.

Received by the editors December 16, 1980.

1980 Mathematics Subject Classification. Primary 55P15; Secondary 54E60, 20E06.

Key words and phrases. Finitely dominated CW complexes, free products with amalgamation. 
2. Proof of Theorem 1.1. We need the following proposition.

2.1. Proposition. Suppose $G$ is a subgroup of a group $H$ and $A$ is a $G$ module. If $Z[H] \otimes_{G} A$ is a projective (finitely generated) $H$ module, where for the tensor product $Z[H]$ has the right $G$ module structure given by $G \hookrightarrow H$, then $A$ is projective (finitely generated).

Proof (DUE TO THE REFEREE). The statement about projective modules is obvious. Although the part concerning finite generation is well known, no convenient proof is in the literature and a proof is therefore given.

Since $G$ is a subgroup of $H, Z[H]$ is a free right $Z[G]$ module. In fact, if $\left\{t_{\lambda} \in H \mid \lambda \in \Lambda\right\}$ is a transversal (i.e. a set of $H / G$ coset representatives) for $G$ in $H$, then $\left\{t_{\lambda} \mid \lambda \in \Lambda\right\}$ is a $Z[G]$ basis for $Z[H]$. Without loss of generality, we may assume that the identity coset $1 G$ is represented by $1 \in H$. It follows easily that if $M$ is any left $Z[G]$ module, then, as $Z[G]$ modules, $Z[H] \otimes_{G} M=\Sigma_{\lambda \in \Lambda} t_{\lambda} M$ where one $t_{\lambda}=1$ (i.e. $M$ is a $Z[G]$ direct summand of $Z[H] \otimes_{G} M$ ).

Now, let $a_{1}, \ldots, a_{k} \in Z[H] \otimes_{G} A$ be a finite set of $Z[H]$ generators. Then each $a_{i}$ can be written as a tuple $a_{i}=\left(a_{i}^{\lambda}\right)(\lambda \in \Lambda)$, where $a_{i}^{\lambda} \in t_{\lambda} A$ for all $\lambda$. In fact, $a_{i}^{\lambda}=t_{\lambda} b_{i}^{\lambda}$, for some $b_{i}^{\lambda} \in A$ and $b_{i}^{\lambda} \neq 0$, for only finitely many $\lambda$. Let $B$ be the $Z[G]$ submodule of $A$ generated by $\left\{b_{i}^{\lambda} \mid i=1, \ldots, k, \lambda \in \Lambda\right\}$. Then $B$ is finitely generated and the inclusion $i: B \rightarrow A$ induces an isomorphism $1 \otimes i: Z[H] \otimes_{G} B$ $\rightarrow Z[H] \otimes_{G} A$. Since

$$
Z[H] \otimes_{G} B \stackrel{1 \otimes_{i}}{\rightarrow} Z[H] \otimes_{G} A \rightarrow Z[H] \otimes_{G}(A / B) \rightarrow 0
$$

is exact, it follows that $Z[H] \otimes_{G}(A / B)=0$. Since $A / B$ is a direct summand of this module, it follows that $A / B=0$ and that $A$ is finitely generated.

Now, the proof of Theorem 1.1 is divided into three cases.

Case 1. Assume: 1. $X_{0}$ is connected, 2. $X_{0}$ is homotopy equivalent to a finite $\mathrm{CW}$ complex $K_{0}$ and 3. $X$ is homotopy equivalent to a finite CW complex $K$.

Let $f_{0}: K_{0} \rightarrow X_{0}$ be a homotopy equivalence. For each $i=1,2$ we can extend $f_{0}$ to $f_{i}^{\prime}: K_{i}^{\prime} \rightarrow X_{i}$ such that $f_{i}^{\prime}$ induces an epimorphism of fundamental groups and $K_{i}^{\prime}$ is the wedge of $K_{0}$ and a finite number of circles. By Lemma 3.11 in [4, p. 18], the kernel of $\pi_{1}\left(f_{i}^{\prime}\right)$ can be expressed as the normal closure of a finite set of elements of $\pi_{1}\left(K_{i}^{\prime}\right)$. Therefore, by attaching a finite number of 2-cells to $K_{i}^{\prime}$ we can form a finite CW complex $K_{i}$ and an extension $f_{i}: K_{i} \rightarrow X_{i}$ of $f_{0}$, inducing an isomorphism of fundamental groups. Thus the following proposition $\mathbf{P}_{m}$ holds for $m=1$.

$\mathrm{P}_{m}$ : There exists a finite CW complex $L_{m}$ with $\operatorname{dim} L_{m} \leqslant \max \left(m, 2+\operatorname{dim} K_{0}\right)$ that is a union of subcomplexes $K_{1}$ and $K_{2}$ with intersection equal to $K_{0}$, and a map $f: L_{m} \rightarrow X$ so that, restricted to $K_{k}, k=0,1,2, f$ gives a map $f_{k}: K_{k} \rightarrow X_{k}$ which is $m$-connected for $k=1,2$, a homotopy equivalence for $k=0$ and each $\pi_{1}\left(f_{k}\right)$, $k=1,2$, is an isomorphism.

Suppose for induction that $\mathbf{P}_{m-1}$ holds, $m \geqslant 2$, and consider the exact sequence

$$
\begin{aligned}
0 \rightarrow C_{*}\left(\bar{M}\left(f_{0}\right), \bar{K}_{0}\right) & \rightarrow C_{*}\left(\bar{M}\left(f_{1}\right), \bar{K}_{1}\right) \\
& \oplus C_{*}\left(\bar{M}\left(f_{2}\right), \bar{K}_{2}\right) \rightarrow C_{*}\left(\tilde{M}(f), \tilde{L}_{m-1}\right) \rightarrow 0,
\end{aligned}
$$


where $\bar{S}=p^{-1}(S), p: \tilde{M}(f) \rightarrow M(f)$ being the universal covering projection of the mapping cylinder $M(f)$ of $f: L_{m-1} \rightarrow X$ and $C_{*}(Y, Z)$ the chain complex of a $C W$ pair $(Y, Z)$.

Since $f_{0}: K_{0} \rightarrow X_{0}$ is a homotopy equivalence, so is $\bar{K}_{0} \rightarrow \bar{M}\left(f_{0}\right)$. Thus, $H_{*}\left(\bar{M}\left(f_{0}\right), \bar{K}_{0}\right)=0$ and we have an isomorphism of $\pi_{1}(X)$ modules $H_{n}\left(\bar{M}\left(f_{1}\right), \bar{K}_{1}\right)$ $\oplus H_{n}\left(\bar{M}\left(f_{2}\right), \bar{K}_{2}\right)$ and $H_{n}\left(\tilde{M}(f), \tilde{L}_{m-1}\right)$ for $n \geqslant 0 . H_{m}\left(\tilde{M}(f), \tilde{L}_{m-1}\right)$ is the first nonvanishing homology group of $\left(\tilde{M}(f), \tilde{L}_{m-1}\right)$ and, by Lemma 4.6 in [4] (see also [5, Theorem A]), it is finitely generated. Hence, both $H_{m}\left(\bar{M}\left(f_{i}\right), \overline{K_{i}}\right), i=1,2$, are finitely generated $\pi_{1}(X)$ modules.

It is well known (see [4, Lemma 6.7]) that

$$
C_{*}\left(\bar{M}\left(f_{i}\right), \overline{K_{i}}\right)=Z\left[\pi_{1}(X)\right] \otimes C_{*}\left(\tilde{M}\left(f_{i}\right), \tilde{K}_{i}\right)
$$

where the tensor product is over $Z\left[\pi_{1}\left(X_{i}\right)\right]$ and $\tilde{M}\left(f_{i}\right), \tilde{K}_{i}$ denote universal covers. Since $\pi_{1}\left(X_{i}\right) \rightarrow \pi_{1}(X)$ is monomorphic, $Z\left[\pi_{1}(X)\right]$ is a free $Z\left[\pi_{1}\left(X_{i}\right)\right]$ module and thus tensoring with it is an exact functor. Hence,

$$
H_{m}\left(\bar{M}\left(f_{i}\right), \overline{K_{i}}\right)=Z\left[\pi_{1}(X)\right] \otimes H_{m}\left(\tilde{M}\left(f_{i}\right), \tilde{K}_{i}\right)
$$

where again the tensor product is over $Z\left[\pi_{1}\left(X_{i}\right)\right]$. By Proposition $2.1, H_{m}\left(\tilde{M}\left(f_{i}\right), \tilde{K_{i}}\right)$ is a finitely generated $\pi_{1}\left(X_{i}\right)$ module, $i=1,2$. As in [5] (see the proof of Theorem A) we can obtain $L_{m}$ and $f^{\prime}: L_{m} \rightarrow X$ by attaching a finite number of $m$-cells to $K_{1}$ and $K_{2}$ and then extending $f$ onto $L_{m}$. This completes the induction.

The proof that $X_{1}, X_{2} \in \mathscr{D}$ is completed as follows. Take an $(m-1)$-connected map $f: L \rightarrow X$ as in $P_{m-1}$, where $m \geqslant 1+\max \left(\operatorname{dim} K_{0}, \operatorname{dim} K, 2\right)$. Then $H_{*}(\tilde{M}(f), \tilde{L})$ is finitely generated, projective and concentrated in dimension $m$ (see [5, Theorem A and Lemma 2.1]). Since

$$
H_{n}\left(\bar{M}\left(f_{1}\right), \bar{K}_{1}\right) \oplus H_{n}\left(\bar{M}\left(f_{2}\right), \bar{K}_{2}\right) \simeq H_{n}(\tilde{M}(f), \tilde{L})
$$

for each $n$, we infer that $H_{*}\left(\bar{M}\left(f_{i}\right), \bar{K}_{i}\right)$ is finitely generated, projective over $Z\left[\pi_{1}(X)\right]$ and concentrated in dimension $m$ for $i=1,2$. Hence, $H_{*}\left(\tilde{M}\left(f_{i}\right), \tilde{K}_{i}\right)$ is a finitely generated projective over $Z\left[\pi_{1}\left(X_{i}\right)\right]$ and concentrated in dimension $m$ (by Proposition 2.1). It follows from [4, Lemma 6.2] that $X_{1}, X_{2} \in \mathscr{D}$.

Case 2. Assume $X_{0}$ is connected. Then, $X \times S^{1}$ and $X_{0} \times S^{1}$ have the homotopy type of finite complexes (see [2]) and by Case 1 we have, $X_{1} \times S^{1}, X_{2} \times S^{1} \in \mathscr{D}$, which implies $X_{1}, X_{2} \in \mathscr{Q}$.

General case. If $X_{0}$ is not connected, we attach a finite number of 1-cells $e_{1}, \ldots, e_{n}$ to it so that $X_{0}^{\prime}=X_{0} \cup e_{1} \cup \cdots \cup e_{n}$ is connected. By Case 2, both $X_{1}^{\prime}=X_{1} \cup X_{0}^{\prime}$ and $X_{2}^{\prime}=X_{2} \cup X_{0}^{\prime}$ belong to $\mathscr{Q}$. Since $X_{i}$ is a retract of $X_{i}^{\prime}, i=1,2$, we infer $X_{1}, X_{2} \in \mathscr{Q}$.

Remark. Our proof of Theorem 1.1 is a simplified version of the original Siebenmann's proof of Complement 6.6(b) (see [4, pp. 48, 54-56]).

Theorem 1.2 can be proved similarly. 
3. Proof of Theorem 1.3. Theorem 1.3 is basically an algebra theorem and will be derived from the following result.

3.1. TheORem. If the free product $P=\langle G * H: A=B, \varphi\rangle$ with amalgamation is finitely presented and $A$ is finitely presented, then both $G$ and $H$ are finitely presented.

For the notion of the free product $P=\langle G * H: A=B, \varphi\rangle$ (denoted also by $G *{ }_{A} H$ ) of groups $G$ and $H$, amalgamating subgroups $A$ of $G$ and $B$ of $H$ by an isomorphism $\varphi$, see [1, p. 179].

The proof of this theorem depends on two lemmata.

3.2. LEMMA. If $G *_{A} H$ is finitely generated, so are $G$ and $H$.

Proof. Let $\left\{g_{\lambda} \mid \lambda \in L\right\}$ and $\left\{h_{\mu} \mid \mu \in M\right\}$ be generators for $G$ and $H$ respectively. By [1, p. 187], $\left\{g_{\lambda}, h_{\mu} \mid \lambda \in L, \mu \in M\right\}$ generate $G{ }^{*}{ }_{A} H$. Since this group is finitely generated, there exist finitely many of the $g_{\lambda}$ 's and $h_{\mu}$ 's, $g_{1}, \ldots, g_{q}$, $h_{1}, \ldots, h_{r}$ such that these elements generate $G *_{A} H$. But then $g_{1}, \ldots, g_{q}$ generate $G$ and $h_{1}, \ldots, h_{r}$ generate $H$.

Suppose now that $A$ has presentation

$$
\left\langle Z_{1}, \ldots, Z_{s} ; W_{1}\left(Z_{1}, \ldots, Z_{s}\right), \ldots, W_{p}\left(Z_{1}, \ldots, Z_{s}\right)\right\rangle \text {. }
$$

Then $G$ has a presentation

$$
\left\langle X_{1}, \ldots, X_{q}, Z_{1}, \ldots, Z_{s} ; U_{\lambda}\left(X_{i}, Z_{k}\right), W_{1}\left(Z_{1}, \ldots, Z_{s}\right), \ldots, W_{p}\left(Z_{1}, \ldots, Z_{s}\right)\right\rangle \text {, }
$$

where $\lambda \in L, U_{\lambda}\left(X_{i}, Z_{k}\right)$ is a word in (possibly all) the $X_{i}$ 's and $Z_{k}$ 's, and $\left\{X_{i}\right\}$ maps to a finite set of generators $\left\{g_{i}\right\}$ for $G$. Suppose that $H$ is presented similarly as

$$
\left\langle Y_{1}, \ldots, Y_{r}, Z_{1}, \ldots, Z_{s} ; V_{\mu}\left(Y_{j}, Z_{1}\right), W_{1}\left(Z_{1}, \ldots, Z_{s}\right), W_{p}\left(Z_{1}, \ldots, Z_{s}\right)\right\rangle,
$$

where $\mu \in N$.

3.3. LEMMA. $G *{ }_{A} H$ has the presentation

$$
\begin{aligned}
\left\langle X_{1}, \ldots, X_{q}, Y_{1}, \ldots, Y_{r}, Z_{1}, \ldots, Z_{s} ;\right. & \\
& \left.U_{\lambda}\left(X_{i}, Z_{k}\right), V_{\mu}\left(X_{j}, Z_{1}\right), \ldots, W_{p}\left(Z_{1}, \ldots, Z_{s}\right)\right\rangle .
\end{aligned}
$$

Proof. This is well known (see [3]).

Proof of Theorem 3.1. We present $A, G, H$ as above and consider the presentation of $G *{ }_{A} H$ given by 3.3. Since $G *{ }_{A} H$ is finitely presented, it has a presentation on the above generators containing only finitely many of the above relators. Thus $G *{ }_{A} H$ may be presented as

$$
\begin{aligned}
\left\langle X_{1}, \ldots, X_{q}, Y_{1}, \ldots, Y_{r}, Z_{1}, \ldots, Z_{s} ; U_{1}\left(X_{i}, Z_{k}\right), \ldots, U_{m}\left(X_{i}, Z_{k}\right),\right. \\
\left.V_{1}\left(Y_{j}, Z_{1}\right), \ldots, V_{n}\left(Y_{j}, Z_{1}\right), W_{1}\left(Z_{1}, \ldots, Z_{s}\right), \ldots, W_{p}\left(Z_{1}, \ldots, Z_{s}\right)\right\rangle .
\end{aligned}
$$

Let

$$
\begin{aligned}
G^{\prime}=\left\langle X_{1}, \ldots, X_{q}, Z_{1}, \ldots, Z_{s} ; U_{1}\left(X_{i}, Z_{k}\right), \ldots, U_{m}\left(X_{i}, Z_{k}\right),\right. \\
\left.W_{1}\left(Z_{1}, \ldots, Z_{s}\right), \ldots, W_{p}\left(Z_{1}, \ldots, Z_{s}\right)\right\rangle .
\end{aligned}
$$


Then there is an obvious epimorphism $\psi^{\prime}: G^{\prime} \rightarrow G$ that maps the subgroup $A^{\prime} \subset G^{\prime}$ generated by $Z_{1}, \ldots, Z_{s}$ isomorphically onto $A$. Similarly, if $H^{\prime}=$ $\left\langle Y_{1}, \ldots, Y_{r}, Z_{1}, \ldots, Z_{s} ; V_{1}\left(Y_{j}, Z_{1}\right), \ldots, V_{n}\left(Y_{j}, Z_{1}\right), W_{1}\left(Z_{1}, \ldots, Z_{s}\right)\right.$, $\left.W_{p}\left(Z_{1}, \ldots, Z_{s}\right)\right\rangle$, there is an epimorphism $\psi^{\prime \prime}: H^{\prime} \rightarrow H$ that maps the subgroup generated by $Z_{1}, \ldots, Z_{s}$ isomorphically onto $A$. Thus $\psi^{\prime}$ and $\psi^{\prime \prime}$ induce a homomorphism $\psi: G{ }^{\prime} *{ }_{A} H^{\prime} \rightarrow G *{ }_{A} H$. Since the presentation for $G^{\prime} *_{A} H^{\prime}$ is identical with that of $G{ }^{*}{ }_{A} H, \psi$ is an isomorphism. It follows easily that $\psi^{\prime}$ and $\psi^{\prime \prime}$ are isomorphisms and, therefore, that $G$ and $H$ are finitely presented.

Proof of Theorem 1.3. Suppose $X_{0}$ is connected. Then $\pi_{1}(X)$ is the free product of $\pi_{1}\left(X_{1}\right)$ and $\pi_{1}\left(X_{2}\right)$ with amalgamation of

$$
\operatorname{im}\left(\pi_{1}\left(X_{0}\right) \rightarrow \pi_{1}\left(X_{1}\right)\right) \text { and } \operatorname{im}\left(\pi_{1}\left(X_{0}\right) \rightarrow \pi_{1}\left(X_{2}\right)\right)
$$

(see [1, p. 180]). Therefore $\pi_{1}\left(X_{i}\right) \rightarrow \pi_{1}(X)$ is a monomorphism for $i=1,2$ and, by Theorem 3.1, $\pi_{1}\left(X_{1}\right)$ and $\pi_{1}\left(X_{2}\right)$ are finitely presented.

By Theorem 1.1, $X_{1}, X_{2} \in \mathscr{D}$.

If $X_{0}$ is not connected, then we apply the trick used in General Case of the proof of Theorem 1.1.

I am indebted to the referee for supplying me with the proof of Proposition 2.1 and for simplifying the proof of Theorem 3.1.

\section{REFERENCES}

1. R. C. Lyndon and P. E. Schupp, Combinatorial group theory, Springer-Verlag, New York, 1977.

2. M. Mather, Counting homotopy types of manifolds, Topology 4 (1965), 93-94.

3. B. H. Newmann, An essay on free products of groups with amalgamation, Philos. Trans. Roy. Soc. London Ser. A 246 (1954), 503-554.

4. L. C. Siebenmann, The obstruction to finding a boundary for an open manifold of dimension greater than five, Thesis, Princeton University, 1965.

5. C. T. C. Wall, Finiteness conditions for CW complexes, Ann. of Math. (2) 81 (1965), 56-69.

Institute of Mathematics, University of WarsaW, PKIN, IX P., 00-901 Warsaw, Poland 